TRANSACTIONS OF THE

AMERICAN MATHEMATICAL SOCIETY

Volume 355, Number 7, Pages 2905-2923

S 0002-9947(03)03274-4

Article electronically published on March 17, 2003

\title{
CRITERIA FOR LARGE DEVIATIONS
}

\author{
HENRI COMMAN
}

AbStRACT. We give the general variational form of

$$
\lim \sup \left(\int_{X} e^{h(x) / t_{\alpha}} \mu_{\alpha}(d x)\right)^{t_{\alpha}}
$$

for any bounded above Borel measurable function $h$ on a topological space $X$, where $\left(\mu_{\alpha}\right)$ is a net of Borel probability measures on $X$, and $\left(t_{\alpha}\right)$ a net in $] 0, \infty[$ converging to 0 . When $X$ is normal, we obtain a criterion in order to have a limit in the above expression for all $h$ continuous bounded, and deduce new criteria of a large deviation principle with not necessarily tight rate function; this allows us to remove the tightness hypothesis in various classical theorems.

\section{INTRODUCTION}

Let $\mathcal{C}_{b}(X)$ be the set of real-valued bounded continuous functions on a topological space $X,\left(\mu_{\alpha}\right)$ a net of Borel probability measures on $X$, and $\left(t_{\alpha}\right)$ a net in $] 0, \infty[$ converging to 0 . For each $[-\infty,+\infty]$-valued Borel measurable function $h$ on $X$, we write $\mu_{\alpha}^{t_{\alpha}}\left(e^{h / t_{\alpha}}\right)$ for $\left(\int_{X} e^{h(x) / t_{\alpha}} \mu_{\alpha}(d x)\right)^{t_{\alpha}}$, and define $\Lambda(h)=\log \lim \mu_{\alpha}^{t_{\alpha}}\left(e^{h / t_{\alpha}}\right)$ provided the limit exists.

The aim of this paper is to clarify the relation between the existence of $\Lambda(h)$ for all $h \in \mathcal{C}_{b}(X)$, and the one of a large deviation principle for $\left(\mu_{\alpha}\right)$ with powers $\left(t_{\alpha}\right)$. This problem originates from Varadhan's theorem, which states that if $X$ is regular, then such a principle with tight rate function $J$ implies the existence of $\Lambda(h)$ for all $[-\infty,+\infty$ [-valued continuous functions $h$ on $X$ satisfying some tail condition (in particular for all $\left.h \in \mathcal{C}_{b}(X)\right)$, with moreover $\Lambda(h)=\sup _{x \in X}\{h(x)-J(x)\}$. This theorem is a crucial argument in the proofs of all related results; in particular, these also hold under some tightness hypothesis (of the rate function, or of the net $\left(\mu_{\alpha}^{t_{\alpha}}\right)$, i.e., exponential tightness).

We present here a new approach based on a variational representation of the functional $\limsup \mu_{\alpha}^{t_{\alpha}}\left(e^{\cdot / t_{\alpha}}\right)$, and a criterion of existence of $\Lambda(\cdot)$ on $\mathcal{C}_{b}(X)$ when $X$ is normal. This leads to functional as well as set-theoretic large deviation criteria, which allow us to remove the tightness condition in various basic results of the theory; moreover, the proofs are nonstandard since there are no compactness arguments in the entire paper. Notice that all the results work for general nets of measures and powers, and (except for Section 4 and " $(i) \Rightarrow(i i)$ " in Theorem 3.3 where $X$ is assumed to be normal) for a general topological states space.

Received by the editors January 3, 2002 and, in revised form, November 9, 2002.

2000 Mathematics Subject Classification. Primary 60F10.

This work was supported in part by FONDECYT Grant 3010005. 
We begin in Section 2 by stating somewhat unusual equivalent definitions of a large deviation principle (Proposition [2.3) which imply the existence of a minimal rate function.

In Section 3, we prove a general variational form of $\lim \sup \mu_{\alpha}^{t_{\alpha}}\left(e^{h / t_{\alpha}} 1_{Y}\right)$ for any Borel measurable function $h$ on $X$ and any Borel set $Y \subset X$ satisfying the (localized) tail condition of Varadhan's theorem (Theorem 3.1). By the same methods, we obtain a sufficient (and necessary if $X$ is normal) condition for the existence of $\Lambda(h)$ for all $h \in \mathcal{C}_{b}(X)$, in the spirit of the Portmanteau theorem (Theorem 3.3). A generalized version of Varadhan's theorem without any tightness assumption is a direct consequence (Corollary 3.4).

In Section 4, assuming that $X$ is normal, we look for necessary and sufficient conditions that $\Lambda(\cdot)$ (as a functional on $\mathcal{C}_{b}(X)$ ) must satisfy in order to have a large deviation principle. This is obtained in Theorem 4.1, which gives five such conditions; in particular, it states that large deviations occur if and only if $\lim \Lambda\left(h_{i}\right)=\Lambda(h)$ for each increasing net $\left(h_{i}\right)$ in $\mathcal{C}_{b}(X)$ converging pointwise to $h \in \mathcal{C}_{b}(X)$. As corollaries, various basic results hold verbatim without tightness assumptions; this is the case for the equivalence between the Laplace and large deviation principles (Corollary 4.2), and for the variational form of a rate function (4.5); a large deviation principle is characterized as a convergence in a narrow space of set-functions much larger than capacities (Remark 4.5); Corollary 4.3 improves Bryc's theorem by weakening the exponential tightness hypothesis; (4.2) gives the infimum of a rate function $J$ on any closed set in terms of $\liminf \mu_{\alpha}^{t_{\alpha}}(\cdot)\left(\right.$ resp. $\left.\lim \sup \mu_{\alpha}^{t_{\alpha}}(\cdot)\right)$, generalizing a well-known expression of $J$.

\section{Preliminaries}

Let $\mathcal{F}$ (resp. $\mathcal{G}$ ) denotes the set of closed (resp. open) subsets of $X$. For each $[0,+\infty]$-valued function $f$ on $X$ we denote by $\bar{f}$ (resp. $\stackrel{f}{f}$ ) the least upper semicontinuous function on $X$ greater than $f$ (resp. the greatest lower semi-continuous function on $X$ less than $f$ ), and define a map $\gamma_{f}: \mathcal{G} \rightarrow[0,+\infty]$ by $\gamma_{f}(G)=$ $\sup _{x \in G} f(x)$ for all $G \in \mathcal{G}$.

We collect here some characterizations of positive bounded upper semi-continuous functions that we will use in the sequel.

Lemma 2.1. There is a bijection between the set of positive bounded upper semicontinuous functions $f$ on $X$ and the set of maps $\gamma: \mathcal{G} \rightarrow[0,+\infty[$ satisfying

$$
\gamma\left(\bigcup_{i \in I} G_{i}\right)=\sup _{i \in I} \gamma\left(G_{i}\right) \quad \text { for all }\left\{G_{i} ; i \in I\right\} \subset \mathcal{G},
$$

given by the maps

- $\gamma \mapsto f_{\gamma}(x)=\inf _{G \in \mathcal{G}, x \in G} \gamma(G)$ for all $x \in X$,

- $f \mapsto \gamma_{f}$.

Moreover, for each positive bounded function $f$ on $X$, the following properties hold:

(i) $\bar{f}=f_{\gamma_{f}}$;

(ii) $\bar{f}$ is the unique positive upper semi-continuous function $h$ on $X$ satisfying $\gamma_{h}=\gamma_{f}$

(iii) $\sup _{x \in Y} \bar{f}(x)=\inf _{G \supset Y, G \in \mathcal{G}} \gamma_{f}(G) \quad$ for all $Y \subset X$;

(iv) $\bar{f}=\bigvee\left\{g \in\left[0,+\infty\left[{ }^{X}: \gamma_{g}=\gamma_{f}\right\}\right.\right.$. 
Proof. Let $f$ be a positive bounded upper semi-continuous function on $X$. For each $Y \subset X$ we have $\sup _{Y} f \leq \sup _{\left\{f<\sup _{Y} f+\varepsilon\right\}} f \leq \sup _{Y} f+\varepsilon$; since $\left\{f<\sup _{Y} f+\varepsilon\right\}$ is open and contains $Y$, we obtain

$$
\sup _{Y} f=\inf _{G \supset Y, G \in \mathcal{G}} \gamma_{f}(G),
$$

whence $f_{\gamma_{f}}=f$. We now prove that $\gamma_{f_{\gamma}}=\gamma$ for all $\gamma: \mathcal{G} \rightarrow[0,+\infty$ [satisfying (2.1); let $\gamma$ be such a map, $\lambda>0$, and define $G_{\lambda}=\bigcup\{G \in \mathcal{G} ; \gamma(G) \leq \lambda\}$. For all $0 \leq \nu<\lambda$ and for all $x \in G_{\nu}$, we have $f_{\gamma}(x) \leq \gamma\left(G_{\nu}\right) \leq \nu$ so that $\bigcup_{\nu<\lambda} G_{\nu} \subset\left\{f_{\gamma}<\lambda\right\}$. For all $x \in\left\{f_{\gamma}<\lambda\right\}$ there is $G \ni x$ such that $\gamma(G)<\lambda$, and thus $G \subset G_{\nu}$ for some $\nu<\lambda$. This shows that $\left\{f_{\gamma}<\lambda\right\} \subset \bigcup_{\nu<\lambda} G_{\nu}$, and thus $\left\{f_{\gamma}<\lambda\right\}=\bigcup_{\nu<\lambda} G_{\nu}$, which is an open set, so that $f_{\gamma}$ is upper semi-continuous. Let $G \in \mathcal{G}$. Clearly, $\sup _{G} f_{\gamma} \leq \gamma(G)$ (with the convention $\sup _{\emptyset}=0$ ). If $\sup _{G} f_{\gamma}<\gamma(G)$, then $G \subset\left\{f_{\gamma}<\gamma(G)-\varepsilon\right\}$ for some $\varepsilon>0$. Since $f_{\gamma}$ is upper semi-continuous, $\left\{f_{\gamma}<\gamma(G)-\varepsilon\right\}$ is open with $\left\{f_{\gamma}<\gamma(G)-\varepsilon\right\} \subset G_{\gamma(G)-\varepsilon}$, and since $\gamma$ is clearly increasing, we obtain $\gamma(G) \leq \gamma\left(\left\{f_{\gamma}<\gamma(G)-\varepsilon\right\}\right) \leq \gamma\left(G_{\gamma(G)-\varepsilon}\right) \leq \gamma(G)-\varepsilon$, which gives the contradiction. Thus $\gamma(G)=\sup _{G} f_{\gamma}$ for all $G \in \mathcal{G}$ and the first assertion is proved.

Let $f$ be a positive bounded function on $X$. Then $\gamma_{f}$ is bounded and satisfies (2.1), so that $f_{\gamma_{f}}$ is upper semi-continuous with $f_{\gamma_{f}} \geq f$. For all positive bounded upper semi-continuous functions $f_{1} \geq f$, we have $\gamma_{f_{1}} \geq \gamma_{f}$ and so $f_{\gamma_{f_{1}}}=f_{1} \geq f_{\gamma_{f}} \geq$ $f$, which implies $f_{\gamma_{f}}=\bar{f}$. This prove $(i)$. If $h$ is a positive upper semi-continuous function on $X$ satisfying $\gamma_{h}=\gamma_{f}$, then $h$ is bounded and (ii) follows from (i). Let $Y \subset X$. By (2.2) we have $\sup _{Y} \bar{f}=\inf _{G \supset Y, G \in \mathcal{G}} \sup _{G} \bar{f}$, and since $\gamma_{\bar{f}}=\gamma_{f}$ by $(i i)$, (iii) holds. Let $h=\bigvee\left\{g \in\left[0,+\infty\left[{ }^{X}: \gamma_{g}=\gamma_{f}\right\}\right.\right.$. It is easy to see that $\gamma_{h}=\gamma_{f}$, and since $f_{\gamma_{h}}$ is upper semi-continuous with $f_{\gamma_{h}} \geq h$, we have $\gamma_{h}=\gamma_{\gamma_{\gamma_{h}}}=\gamma_{f}$ whence $h=f_{\gamma_{h}}=\bar{f}$ by $(i)$. Thus (iv) holds.

Definition 2.2. We say that $\left(\mu_{\alpha}\right)$ satisfies a large deviation principle with powers $\left(t_{\alpha}\right)$ if there is a lower semi-continuous function $J: X \rightarrow[0, \infty]$ such that

$$
\limsup \mu_{\alpha}^{t_{\alpha}}(F) \leq \sup _{x \in F} e^{-J}(x) \leq \sup _{x \in G} e^{-J}(x) \leq \liminf \mu_{\alpha}^{t_{\alpha}}(G)
$$

for all $F \in \mathcal{F}, G \in \mathcal{G}$ with $F \subset G$. Then, $J$ is called a rate function for $\left(\mu_{\alpha}^{t_{\alpha}}\right)$, which is said to be tight if it has compact level sets.

Notice that in the literature, a large deviation principle is in general defined for nets $\left(\mu_{\epsilon}^{\epsilon}\right)_{\epsilon>0}$ or sequences $\left(\mu_{n}^{1 / n}\right)_{n \in \mathbf{N}^{*}}$. In the sequel, when we will refer to known results that will be proved again, we will not make this distinction and state them with general nets of measures and powers.

By (2.3), the following proposition shows that the set of rate functions for $\left(\mu_{\alpha}^{t_{\alpha}}\right)$ has a minimal element; it is the only one if $X$ is regular since it is well known ([2], Lemma 4.1.4).

Proposition 2.3. The following statements are equivalent:

(i) $\left(\mu_{\alpha}\right)$ satisfies a large deviation principle with powers $\left(t_{\alpha}\right)$.

(ii) There is a map $\gamma: \mathcal{G} \rightarrow[0,1]$ such that

(a) $\lim \sup \mu_{\alpha}^{t_{\alpha}}(F) \leq \gamma(G) \leq \liminf \mu_{\alpha}^{t_{\alpha}}(G)$ for all $F \in \mathcal{F}, G \in \mathcal{G}$ with $F \subset G$.

(b) $\gamma\left(\bigcup_{i \in I} G_{i}\right)=\sup _{i \in I} \gamma\left(G_{i}\right) \quad$ for all $\left\{G_{i}: i \in I\right\} \subset \mathcal{G}$. 
(iii) There is a function $f: X \rightarrow[0,1]$ such that

$$
\limsup \mu_{\alpha}^{t_{\alpha}}(F) \leq \sup _{x \in G} f(x) \leq \liminf \mu_{\alpha}^{t_{\alpha}}(G)
$$

for all $F \in \mathcal{F}, G \in \mathcal{G}$ with $F \subset G$.

(iv) There is a function $f: X \rightarrow[0,1]$ such that

$$
\limsup \mu_{\alpha}^{t_{\alpha}}(F) \leq \sup _{x \in F} f(x) \leq \liminf \mu_{\alpha}^{t_{\alpha}}(G)
$$

for all $F \in \mathcal{F}, G \in \mathcal{G}$ with $F \subset G$.

If (i) holds, then (i) holds with rate function $J$ given by

$$
\left.e^{-J}=\bigvee\left\{f \in[0,1]^{X}: f \text { satisfies (iii) (resp. }(i v)\right)\right\},
$$

and

$$
\gamma_{e^{-J}}=\bigvee\left\{\gamma \in[0,1]^{\mathcal{G}}: \gamma \text { satisfies }(i i)\right\} .
$$

If (ii) holds with $\gamma$, then (i) holds with rate function $-\log f_{\gamma}$ where

$$
f_{\gamma}(x)=\inf _{G \in \mathcal{G}, x \in G} \gamma(G) \quad \text { for all } x \in X .
$$

If (iii) (resp. (iv)) holds with $f$, then (i) holds with rate function $-\log \bar{f}$.

Proof. If (ii) holds with $\gamma$, then $f_{\gamma}$ is upper semi-continuous and $(i)$ holds with rate function $-\log f_{\gamma}$ by Lemma 2.1.

If $(i i i)$ holds with $f$, then $(i i)$ holds with $\gamma_{f}$, and so $(i)$ holds with rate function $-\log \bar{f}$, since $f_{\gamma_{f}}=\bar{f}$ by Lemma 2.1

If (iv) holds with $f$, then put $\gamma(G)=\sup _{F \subset G} \sup _{F} f$ for all $G \in \mathcal{G}$, and notice that $\gamma$ satisfies $(i i)$. Thus $(i)$ holds with rate function $-\log f_{\gamma}$. Since $f \leq \bar{f} \leq f_{\gamma}$, we have

$$
\sup _{F} f \leq \sup _{F} \bar{f} \leq \sup _{G} \bar{f}=\sup _{G} f \leq \sup _{G} f_{\gamma}
$$

for all $F \in \mathcal{F}, G \in \mathcal{G}$ with $F \subset G$ (the equality follows from Lemma 2.1 (iii)). Thus $(i)$ holds with rate function $-\log \bar{f}$.

If $(i)$ holds, then (ii), (iii) and (iv) hold. The function

$$
\left.h=\bigvee\left\{f \in[0,1]^{X}: f \text { satisfies (iii) (resp. }(i v)\right)\right\}
$$

obviously satisfies (iii) (resp. (iv)); the same for $\bar{h}$ by the preceding discussion, and $h=\bar{h}$ by the definition of $h$; put $e^{-J}=\bar{h}$ and obtain (2.3). The map $\gamma_{M}=\bigvee\{\gamma \in$ $[0,1]^{\mathcal{G}}: \gamma$ satisfies $\left.(i i)\right\}$ satisfies $(i i)$, and so $(i)$ holds with rate function $J$ given by $e^{-J}=f_{\gamma_{M}}$. Since $\gamma_{e^{-J}}=\gamma_{\gamma_{\gamma_{M}}}=\gamma_{M}$, (2.4) holds.

Corollary 2.4. (Contraction principle) Let $Y$ be a topological space, and $\pi: X \rightarrow$ $Y$ a continuous function. If $\left(\mu_{\alpha}\right)$ satisfies a large deviation principle with powers $\left(t_{\alpha}\right)$ and rate function $J^{X}$, then $\left(\pi\left[\mu_{\alpha}\right]\right)$ satisfies a large deviation principle with powers $\left(t_{\alpha}\right)$ and rate function $J^{Y}=\stackrel{\circ}{l}$ where $l(y)=\inf _{x \in \pi^{-1}(y)} J^{X}(x)$ for all $y \in Y$.

Proof. Let $J^{X}$ be a rate function for $\left(\mu_{\alpha}^{t_{\alpha}}\right)$. The relations

$$
\begin{aligned}
\limsup \pi\left[\mu_{\alpha}\right]^{t_{\alpha}}(F) & =\limsup \mu_{\alpha}^{t_{\alpha}}\left(\pi^{-1}(F)\right) \\
& \leq \sup _{\pi^{-1}(F)} e^{-J^{X}} \leq \sup _{\pi^{-1}(G)} e^{-J^{X}} \leq \liminf \pi\left[\mu_{\alpha}\right]^{t_{\alpha}}(G)
\end{aligned}
$$


for all $F \in \mathcal{F}, G \in \mathcal{G}$ with $F \subset G$ and Proposition 2.3 show that $\left(\pi\left[\mu_{\alpha}\right]\right)$ satisfies a large deviation principle with powers $\left(t_{\alpha}\right)$ and rate function $J^{Y}=-\log \bar{f}$ where

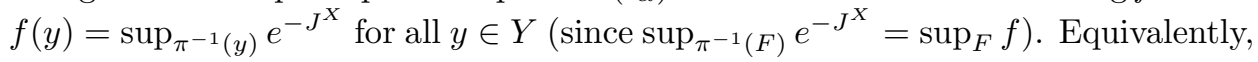
$J^{Y}=\stackrel{\circ}{l}$ where $l(y)=\inf _{\pi^{-1}(y)} J^{X}$ for all $y \in Y$.

\section{A general VARiational Formula}

Up to now, the only known condition that ensures the existence of $\Lambda(h)$ for all $h \in \mathcal{C}_{b}(X)$ (and more generally for all $[-\infty,+\infty$ [-valued continuous functions $h$ on $X$ satisfying the tail condition

$$
\left.\lim _{M \rightarrow \infty} \lim \sup \mu_{\alpha}^{t_{\alpha}}\left(e^{h / t_{\alpha}} 1_{\left\{e^{h}>M\right\}}\right)=0\right)
$$

is the existence of a large deviation principle with tight rate function, and $\Lambda(h)$ is expressed in a variational form in terms of this rate function (Varadhan's theorem, [2] Theorem 4.3.1). In this section, we generalize these results in two directions. First, Theorem 3.1 gives the general variational form of $\lim \sup \mu_{\alpha}^{t_{\alpha}}\left(e^{h / t_{\alpha}} 1_{Y}\right)$ for any Borel set $Y \subset X$ and any Borel measurable function $h$ on $X$ satisfying the localized tail condition (3.2). Next, Theorem 3.3 gives a sufficient condition for the existence of $\Lambda(\cdot)$ on $\mathcal{C}_{b}(X)$ which is also necessary when $X$ is normal; moreover, the variational form of $\Lambda(\cdot)$ is obtained in terms of any set-function $\gamma \in[0,1]^{\mathcal{F} \cup \mathcal{G}}$ satisfying the typical in-between inequalities of large deviations (3.23). As a consequence, Varadhan's theorem is generalized in various ways (Corollary 3.2 and Corollary 3.4).

For each map $h: X \rightarrow[-\infty,+\infty]$ we put $F_{\lambda, \varepsilon}^{h}=\left\{e^{h} \in[\lambda-\varepsilon, \lambda+\varepsilon]\right\}$ and $G_{\lambda, \varepsilon}^{h}=\left\{e^{h} \in\right] \lambda-\varepsilon, \lambda+\varepsilon[\}$ for all $\lambda \geq 0$ and for all $\varepsilon>0$.

Theorem 3.1. For each Borel set $Y \subset X$, and for each $[-\infty,+\infty]$-valued Borel measurable function $h$ on $X$ satisfying

$$
\lim _{M \rightarrow \infty} \lim \sup \mu_{\alpha}^{t_{\alpha}}\left(e^{h / t_{\alpha}} 1_{\left\{e^{h}>M\right\} \cap Y}\right)=0,
$$

we have

$$
\begin{gathered}
\limsup \mu_{\alpha}^{t_{\alpha}}\left(e^{h / t_{\alpha}} 1_{Y}\right)=\sup _{\lambda \geq 0, \varepsilon>0}\left\{(\lambda-\varepsilon) \lim \sup \mu_{\alpha}^{t_{\alpha}}\left(F_{\lambda, \varepsilon}^{h} \cap Y\right)\right\} \\
=\sup _{\left\{x \in Y, \varepsilon>0: e^{h(x)} \leq M\right\}}\left\{\left(e^{h(x)}-\varepsilon\right) \lim \sup \mu_{\alpha}^{t_{\alpha}}\left(G_{e^{h(x)}, \varepsilon}^{h} \cap Y\right)\right\}
\end{gathered}
$$

for some $M \in\left[0,+\infty\left[\right.\right.$. Moreover, $\lim \mu_{\alpha}^{t_{\alpha}}\left(e^{h / t_{\alpha}} 1_{Y}\right)$ exists if

$$
\sup _{\lambda \geq 0, \varepsilon>0}\left\{(\lambda-\varepsilon) \liminf \mu_{\alpha}^{t_{\alpha}}\left(F_{\lambda, \varepsilon}^{h} \cap Y\right)\right\}=\sup _{\lambda \geq 0, \varepsilon>0}\left\{(\lambda-\varepsilon) \limsup \mu_{\alpha}^{t_{\alpha}}\left(F_{\lambda, \varepsilon}^{h} \cap Y\right)\right\} .
$$

Proof. Let $Y$ be any Borel subset of $X$. Put $g=e^{h}, G_{\lambda, \varepsilon}=G_{\lambda, \varepsilon}^{h} \cap Y, F_{\lambda, \varepsilon}=F_{\lambda, \varepsilon}^{h} \cap Y$ for all $\lambda \geq 0$ and for all $\varepsilon>0$. We have

$$
\begin{aligned}
\limsup \mu_{\alpha}^{t_{\alpha}}\left(g^{1 / t_{\alpha}} 1_{Y}\right) & \geq \limsup \mu_{\alpha}^{t_{\alpha}}\left(g^{1 / t_{\alpha}} 1_{F_{\lambda, \varepsilon}}\right) \\
& \geq(\lambda-\varepsilon) \lim \sup \mu_{\alpha}^{t_{\alpha}}\left(F_{\lambda, \varepsilon}\right)
\end{aligned}
$$

for all $\lambda \geq 0$ and for all $\varepsilon>0$, and so

$$
\limsup \mu_{\alpha}^{t_{\alpha}}\left(g^{1 / t_{\alpha}} 1_{Y}\right) \geq \sup _{\lambda \geq 0, \varepsilon>0}\left\{(\lambda-\varepsilon) \lim \sup \mu_{\alpha}^{t_{\alpha}}\left(F_{\lambda, \varepsilon}\right)\right\} .
$$


Thus, in order to prove (3.3) and (3.4), we have to prove that for some $M<\infty$,

$$
\limsup \mu_{\alpha}^{t_{\alpha}}\left(g^{1 / t_{\alpha}} 1_{Y}\right) \leq \sup _{\{x \in Y, \varepsilon>0: g(x) \leq M\}}\left\{(g(x)-\varepsilon) \limsup \mu_{\alpha}^{t_{\alpha}}\left(G_{g(x), \varepsilon}\right)\right\} .
$$

For all $M \geq 0$, for all $N \in \mathbf{N}^{*}$ and for all $1 \leq j \leq N$, we define

$$
F_{M, N, j}=\{g \in[(j-1) M / N, j M / N]\} \cap Y .
$$

We have

$$
\begin{gathered}
\limsup \mu_{\alpha}^{t_{\alpha}}\left(g^{1 / t_{\alpha}} 1_{Y}\right) \leq \limsup \left(\sum_{j=1}^{N} \mu_{\alpha}\left(g^{1 / t_{\alpha}} 1_{F_{M, N, j}}\right)+\mu_{\alpha}\left(g^{1 / t_{\alpha}} 1_{\{g>M\} \cap Y}\right)\right)^{t_{\alpha}} \\
\quad \leq \max _{1 \leq j \leq N} \limsup \mu_{\alpha}^{t_{\alpha}}\left(g^{1 / t_{\alpha}} 1_{F_{M, N, j}}\right) \vee \limsup \mu_{\alpha}^{t_{\alpha}}\left(g^{1 / t_{\alpha}} 1_{\{g>M\} \cap Y}\right) .
\end{gathered}
$$

Since

$$
\limsup \mu_{\alpha}^{t_{\alpha}}\left(g^{1 / t_{\alpha}} 1_{F_{M, N, j}}\right) \leq \lim \sup \mu_{\alpha}^{t_{\alpha}}\left(F_{M, N, j}\right)\left\|g 1_{F_{M, N, j}}\right\|,
$$

it follows from (3.7) that

$$
\begin{gathered}
\limsup \mu_{\alpha}^{t_{\alpha}}\left(g^{1 / t_{\alpha}} 1_{Y}\right) \\
\leq \max _{1 \leq j \leq N}\left\|g 1_{F_{M, N, j}}\right\| \limsup \mu_{\alpha}^{t_{\alpha}}\left(F_{M, N, j}\right) \vee \lim \sup \mu_{\alpha}^{t_{\alpha}}\left(g^{1 / t_{\alpha}} 1_{\{g>M\} \cap Y}\right) .
\end{gathered}
$$

Let $M \rightarrow \infty, N \rightarrow \infty$ in (3.8) and use (3.2) to obtain some $M_{0} \in[0, \infty[$ such that

$$
\limsup \mu_{\alpha}^{t_{\alpha}}\left(g^{1 / t_{\alpha}} 1_{Y}\right) \leq \liminf _{N \rightarrow \infty} \max _{1 \leq j \leq N}\left\{\left\|g 1_{F_{M_{0}, N, j}}\right\| \lim \sup \mu_{\alpha}^{t_{\alpha}}\left(F_{M_{0}, N, j}\right)\right\} .
$$

Thus, to obtain (3.6) it suffices to show

$$
\begin{aligned}
& \liminf _{N \rightarrow \infty} \max _{1 \leq j \leq N}\left\{\left\|g 1_{F_{M_{0}, N, j}}\right\| \limsup \mu_{\alpha}^{t_{\alpha}}\left(F_{M_{0}, N, j}\right)\right\} \\
\leq & \sup _{\left\{x \in Y, \varepsilon>0: g(x) \leq M_{0}\right\}}\left\{(g(x)-\varepsilon) \lim \sup \mu_{\alpha}^{t_{\alpha}}\left(G_{g(x), \varepsilon}\right)\right\} .
\end{aligned}
$$

If (3.10) does not hold, then there exists $\nu>0$ such that

$$
\begin{gathered}
\liminf _{N \rightarrow \infty} \max _{1 \leq j \leq N}\left\{\left\|g 1_{F_{M_{0}, N, j}}\right\| \lim \sup \mu_{\alpha}^{t_{\alpha}}\left(F_{M_{0}, N, j}\right)\right\} \\
\left.>\sup _{\left\{x \in Y, \varepsilon>0: g(x) \leq M_{0}\right\}}\left\{(g(x)+\nu-\varepsilon) \lim \sup \mu_{\alpha}^{t_{\alpha}}\left(G_{g(x), \varepsilon}\right)\right\}\right) .
\end{gathered}
$$

Take $0<\varepsilon_{0}<\nu / 2$ in (3.11) and obtain

$$
\begin{gathered}
\liminf _{N \rightarrow \infty} \max _{1 \leq j \leq N}\left\{\left\|g 1_{F_{M_{0}, N, j}}\right\| \lim \sup \mu_{\alpha}^{t_{\alpha}}\left(F_{M_{0}, N, j}\right)\right\} \\
>\sup _{\left\{x \in Y ; g(x) \leq M_{0}\right\}}\left\{\left(g(x)+\varepsilon_{0}\right) \lim \sup \mu_{\alpha}^{t_{\alpha}}\left(G_{g(x), \varepsilon}\right)\right\} .
\end{gathered}
$$

But for all $0 \leq \lambda \leq M_{0}$ and for all $N>M_{0} / \varepsilon_{0}$ we have

$$
\left(\lambda+\varepsilon_{0}\right) \lim \sup \mu_{\alpha}^{t_{\alpha}}\left(G_{\lambda, \varepsilon_{0}}\right) \geq\left\|g 1_{F_{M_{0}, N, j_{\lambda}}}\right\| \lim \sup \mu_{\alpha}^{t_{\alpha}}\left(F_{M_{0}, N, j_{\lambda}}\right)
$$

where $j_{\lambda}$ is such that $\lambda \in\left[\left(j_{\lambda}-1\right) M_{0} / N, j_{\lambda} M_{0} / N\right]\left(\right.$ since $\left[\left(j_{\lambda}-1\right) M_{0} / N, j_{\lambda} M_{0} / N\right] \subset$ ]$\lambda-\varepsilon_{0}, \lambda+\varepsilon_{0}\left[\right.$ ). When $\lambda$ ranges over $\left[0, M_{0}\right], j_{\lambda}$ ranges over $\{j: 1 \leq j \leq N\}$, and (3.13) implies

$$
\begin{gathered}
\sup _{0 \leq \lambda \leq M_{0}}\left\{\left(\lambda+\varepsilon_{0}\right) \limsup \mu_{\alpha}^{t_{\alpha}}\left(G_{\lambda, \varepsilon_{0}}\right)\right\} \\
\geq \max _{1 \leq j \leq N}\left\{\left\|g 1_{F_{M_{0}, N, j}}\right\| \lim \sup \mu_{\alpha}^{t_{\alpha}}\left(F_{M_{0}, N, j}\right)\right\}
\end{gathered}
$$


for all $N>M_{0} / \varepsilon_{0}$. Notice that for all $N \in \mathbf{N}^{*}$ and for all $1 \leq j \leq N$, if $F_{M_{0}, N, j} \neq \emptyset$, then $j=j_{g(x)}$ for some $x \in Y$. Thus it suffices to consider $\lambda \in\{g(x): x \in Y, g(x) \leq$ $\left.M_{0}\right\}$ in the L.H.S. of (3.14), that is,

$$
\begin{aligned}
& \sup _{\left\{x \in Y: g(x) \leq M_{0}\right\}}\left\{\left(g(x)+\varepsilon_{0}\right) \lim \sup \mu_{\alpha}^{t_{\alpha}}\left(G_{g(x), \varepsilon_{0}}\right)\right\} \\
& \geq \max _{1 \leq j \leq N}\left\{\left\|g 1_{F_{M_{0}, N, j}}\right\| \lim \sup \mu_{\alpha}^{t_{\alpha}}\left(F_{M_{0}, N, j}\right)\right\}
\end{aligned}
$$

for all $N>M_{0} / \varepsilon_{0}$, which contradicts (3.12); it follows that (3.10), 3.6), and finally (3.3) and (3.4) hold. In the same way that we obtained (3.5), we have

$$
\liminf \mu_{\alpha}^{t_{\alpha}}\left(g^{1 / t_{\alpha}} 1_{Y}\right) \geq \sup _{\lambda \geq 0, \varepsilon>0}\left\{(\lambda-\varepsilon) \liminf \mu_{\alpha}^{t_{\alpha}}\left(F_{\lambda, \varepsilon}\right)\right\}
$$

and the last assertion follows from (3.4).

A localized version of Varadhan's theorem states that if $X$ is regular and if $\left(\mu_{\alpha}\right)$ satisfies a large deviation principle with powers $\left(t_{\alpha}\right)$ and tight rate function $J$, then (3.18) and (3.19) hold with $l=J$ ([2], Exercise 4.3.11). The following corollary removes all the hypotheses on $l$ and $X$.

Corollary 3.2. Let $l$ be $a[0,+\infty]$-valued function on $X$ satisfying

$$
\begin{gathered}
\forall F \in \mathcal{F}, \quad \lim \sup \mu_{\alpha}^{t_{\alpha}}(F) \leq \sup _{x \in F} e^{-l(x)} \\
\text { (resp. } \left.\forall G \in \mathcal{G}, \quad \liminf \mu_{\alpha}^{t_{\alpha}}(G) \geq \sup _{x \in G} e^{-l(x)}\right) .
\end{gathered}
$$

Then, for each $[-\infty,+\infty]$-valued continuous function $h$ on $X$ satisfying (3.1), we have

$$
\begin{aligned}
& \forall F \in \mathcal{F}, \quad \lim \sup \mu_{\alpha}^{t_{\alpha}}\left(e^{h / t_{\alpha}} 1_{F}\right) \leq \sup _{x \in F, h(x)<\infty} e^{h(x)} e^{-l(x)} \\
& \text { (resp. } \left.\forall G \in \mathcal{G}, \quad \liminf \mu_{\alpha}^{t_{\alpha}}\left(e^{h / t_{\alpha}} 1_{G}\right) \geq \sup _{x \in G, h(x)<\infty} e^{h(x)} e^{-l(x)}\right) .
\end{aligned}
$$

Proof. Suppose that (3.16) holds and (3.18) does not hold for some $[-\infty,+\infty]-$ valued continuous function $h$ on $X$ satisfying (3.1). Since

$$
\limsup \mu_{\alpha}^{t_{\alpha}}\left(e^{h / t_{\alpha}} 1_{F}\right)=\sup _{\{x \in F, \varepsilon>0: h(x)<\infty\}}\left\{\left(e^{h(x)}-\varepsilon\right) \limsup \mu_{\alpha}^{t_{\alpha}}\left(F_{e^{h(x)}, \varepsilon}^{h} \cap F\right)\right\}
$$

for all $F \in \mathcal{F}$ by Theorem 3.1, there exists $F_{0} \in \mathcal{F}, x_{0} \in F_{0}$ with $h\left(x_{0}\right)<\infty$, and $\varepsilon_{0}>0$ such that

$$
\left(e^{h\left(x_{0}\right)}-\varepsilon_{0}\right) \limsup \mu_{\alpha}^{t_{\alpha}}\left(F_{e^{h\left(x_{0}\right)}, \varepsilon_{0}}^{h} \cap F\right)>\sup _{x \in F, h(x)<\infty} e^{h(x)} e^{-l(x)} .
$$

By (3.16) we have

$$
\left(e^{h\left(x_{0}\right)}-\varepsilon_{0}\right) \sup _{x \in F_{e^{h\left(x_{0}\right)}, \varepsilon_{0}}^{h} \cap F} e^{-l(x)}>\sup _{x \in F, h(x)<\infty} e^{h(x)} e^{-l(x)},
$$

and so there exists $x_{1} \in F_{e^{h\left(x_{0}\right)}, \varepsilon_{0}}^{h} \cap F$ such that

$$
\left(e^{h\left(x_{0}\right)}-\varepsilon_{0}\right) e^{-l\left(x_{1}\right)}>\sup _{x \in F, h(x)<\infty} e^{h(x)} e^{-l(x)} .
$$

Since $e^{h\left(x_{1}\right)} \geq e^{h\left(x_{0}\right)}-\varepsilon_{0}$ we obtain

$$
e^{h\left(x_{1}\right)} e^{-l\left(x_{1}\right)}>\sup _{x \in F, h(x)<\infty} e^{h(x)} e^{-l(x)}
$$


with $x_{1} \in F$ and $h\left(x_{1}\right)<\infty$, whence the contradiction. Suppose now that (3.17) holds and (3.19) does not hold for some $[-\infty,+\infty]$-valued continuous function $h$ on $X$ satisfying (3.1). By (3.15), there exists $G_{0} \in \mathcal{G}$ such that

$$
\begin{gathered}
\sup _{x \in G_{0}, h(x)<\infty} e^{h(x)} e^{-l(x)}>\liminf \mu_{\alpha}^{t_{\alpha}}\left(e^{h / t_{\alpha}} 1_{G_{0}}\right) \\
\geq \sup _{\left\{x \in G_{0}, \varepsilon>0: h(x)<\infty\right\}}\left\{\left(e^{h(x)}-\varepsilon\right) \liminf \mu_{\alpha}^{t_{\alpha}}\left(G_{e^{h(x)}, \varepsilon}^{h} \cap G_{0}\right)\right\} .
\end{gathered}
$$

Thus, there exists $x_{0} \in G_{0}$ with $h\left(x_{0}\right)<\infty$, and $\nu>0$ such that

$$
e^{h\left(x_{0}\right)} e^{-l\left(x_{0}\right)}>\nu+\sup _{\left\{x \in G_{0}, \varepsilon>0: h(x)<\infty\right\}}\left\{\left(e^{h(x)}-\varepsilon\right) \liminf \mu_{\alpha}^{t_{\alpha}}\left(G_{e^{h(x)}, \varepsilon}^{h} \cap G_{0}\right)\right\}
$$

and

$$
e^{h\left(x_{0}\right)} e^{-l\left(x_{0}\right)}>\sup _{\left\{x \in G_{0}, \varepsilon>0: h(x)<\infty\right\}}\left\{\left(e^{h(x)}-\varepsilon+\nu\right) \liminf \mu_{\alpha}^{t_{\alpha}}\left(G_{e^{h(x)}, \varepsilon}^{h} \cap G_{0}\right)\right\} .
$$

By taking $x=x_{0}$ and $\varepsilon_{0}<\nu$ in the R. H. S. of (3.20) we have

$$
\left.e^{h\left(x_{0}\right)} e^{-l\left(x_{0}\right)}>e^{h\left(x_{0}\right)} \liminf \mu_{\alpha}^{t_{\alpha}}\left(G_{e^{h\left(x_{0}\right)}, \varepsilon_{0}}^{h} \cap G_{0}\right)\right\},
$$

and by (3.17)

$$
e^{h\left(x_{0}\right)} e^{-l\left(x_{0}\right)}>e^{h\left(x_{0}\right)} \sup _{x \in G_{e^{h\left(x_{0}\right)}, \varepsilon_{0}}^{h} \cap G} e^{-l(x)},
$$

which gives the contradiction.

A direct consequence of Corollary 3.2 is that Varadhan's theorem can be stated verbatim for a general state space and with any function (in place of a tight rate function) $l: X \rightarrow[0,+\infty]$ satisfying the large deviations lower and upper bounds:

$$
\limsup \mu_{\alpha}^{t_{\alpha}}(F) \leq \sup _{x \in F} e^{-l(x)} \leq \sup _{x \in G} e^{-l(x)} \leq \liminf \mu_{\alpha}^{t_{\alpha}}(G)
$$

for all $F \in \mathcal{F}, G \in \mathcal{G}$ with $F \subset G$; that is to say, $\Lambda(h)$ exists and

$$
\Lambda(h)=\sup _{x \in X, h(x)<\infty}\{h(x)-l(x)\}
$$

for all $[-\infty,+\infty]$-valued continuous functions $h$ on $X$ satisfying (3.1). We will see with Corollary 3.4 that it is possible to go further in the generalization of Varadhan's theorem obtaining the same conclusions with hypothesis weaker than (3.21).

Recall that $X$ is normal if and only if the following interpolation property holds: if $f$ and $g$ are real-valued respectively upper and lower semi-continuous functions on $X$ such that $f \leq g$, then there is a continuous function $h$ on $X$ satisfying $f \leq h \leq g$.

Theorem 3.3. Consider the following statements:

(i) $\Lambda(h)$ exists for all $h \in \mathcal{C}_{b}(X)$;

(ii) $\limsup \mu_{\alpha}^{t_{\alpha}}(F) \leq \liminf \mu_{\alpha}^{t_{\alpha}}(G)$ for all $F \in \mathcal{F}, G \in \mathcal{G}$ with $F \subset G$.

If $X$ is normal, then $(i) \Rightarrow($ ii). If (ii) holds, then (i) holds and moreover for each $[-\infty,+\infty]$-valued continuous function $h$ on $X$ satisfying (3.1) we have for some $M \in[0,+\infty[$,

$\left.(3.22) e^{\Lambda(h)}=\sup _{\lambda \geq 0, \varepsilon>0}\left\{(\lambda-\varepsilon) \gamma\left(F_{\lambda, \varepsilon}^{h}\right)\right\}=\sup _{\left\{x \in X, \varepsilon>0: e^{h(x)} \leq M\right\}}\left\{\left(e^{h(x)}-\varepsilon\right) \gamma\left(G_{e^{h(x)}, \varepsilon}^{h}\right)\right\}\right)$

for all maps $\gamma: \mathcal{F} \cup \mathcal{G} \rightarrow[0,1]$ satisfying

$$
\limsup \mu_{\alpha}^{t_{\alpha}}(F) \leq \gamma(F) \leq \gamma(G) \leq \liminf \mu_{\alpha}^{t_{\alpha}}(G)
$$


for all $F \in \mathcal{F}, G \in \mathcal{G}$ with $F \subset G$.

Proof. Suppose that $(i)$ holds and $X$ is normal. For each $F \in \mathcal{F}$ and $G \in \mathcal{G}$ with $F \subset G$, there exists $h \in \mathcal{C}_{b}(X)$ such that $1_{F} \leq h \leq 1_{G}$. Since $1_{F} \leq e^{n h-n} \leq$ $e^{-n} 1_{X \backslash G} \vee 1_{G}$ for all $n \in N$, we obtain

$$
\begin{aligned}
\limsup \mu_{\alpha}^{t_{\alpha}}(F) & \leq \inf _{n} e^{\Lambda(n h-n)} \\
& \leq \inf _{n} \liminf \left\{e^{-n}+\mu_{\alpha}^{t_{\alpha}}(G)\right\} \leq \liminf \mu_{\alpha}^{t_{\alpha}}(G)
\end{aligned}
$$

and $(i i)$ holds.

Suppose that (ii) holds. Let $h$ be a $[-\infty,+\infty]$-valued continuous function on $X$ satisfying (3.1), and $\gamma: \mathcal{F} \cup \mathcal{G} \rightarrow[0,1]$ satisfying (3.23). Put $g=e^{h}$ and let us use the same notation as in the proof of Theorem 3.1 (with $Y=X$ ). For all $\lambda \geq 0$, for all $\varepsilon>0$ and for all $\delta>0$ with $\delta>\varepsilon$, we have by (3.23),

$$
\begin{gathered}
\liminf \mu_{\alpha}^{t_{\alpha}}\left(g^{1 / t_{\alpha}}\right) \geq \liminf \mu_{\alpha}^{t_{\alpha}}\left(g^{1 / t_{\alpha}} 1_{G_{\lambda, \delta}}\right) \\
\geq(\lambda-\delta) \gamma\left(G_{\lambda, \delta}\right) \geq(\lambda-\delta) \gamma\left(F_{\lambda, \varepsilon}\right) .
\end{gathered}
$$

Thus

$$
\begin{gathered}
\liminf \mu_{\alpha}^{t_{\alpha}}\left(g^{1 / t_{\alpha}}\right) \geq \lim _{\delta \rightarrow \varepsilon}(\lambda-\delta) \gamma\left(F_{\lambda, \varepsilon}\right) \\
\geq(\lambda-\varepsilon) \gamma\left(F_{\lambda, \varepsilon}\right) \geq(\lambda-\varepsilon) \gamma\left(G_{\lambda, \varepsilon}\right)
\end{gathered}
$$

and

$$
\begin{gathered}
\liminf \mu_{\alpha}^{t_{\alpha}}\left(g^{1 / t_{\alpha}}\right) \geq \sup _{\lambda \geq 0, \varepsilon>0}\left\{(\lambda-\varepsilon) \gamma\left(F_{\lambda, \varepsilon}\right)\right\} \\
\geq \sup _{\lambda \geq 0, \varepsilon>0}\left\{(\lambda-\varepsilon) \gamma\left(G_{\lambda, \varepsilon}\right)\right\} .
\end{gathered}
$$

In order to prove (3.22), we have to prove that for some $M<\infty$,

$$
\limsup \mu_{\alpha}^{t_{\alpha}}\left(g^{1 / t_{\alpha}}\right) \leq \sup _{\left\{x \in X, \varepsilon>0: e^{h(x)} \leq M\right\}}\left\{\left(e^{h(x)}-\varepsilon\right) \gamma\left(G_{e^{h(x)}, \varepsilon}\right)\right\} .
$$

By using (3.23), and in the same way that we have obtained (3.10) in the proof of Theorem 3.1] we find some $M_{0} \in[0, \infty$ [ such that to prove (3.24) it suffices to prove $\liminf _{N \rightarrow \infty} \max _{1 \leq j \leq N}\left\{\left\|g 1_{F_{M_{0}, N, j}}\right\| \gamma\left(F_{M_{0}, N, j}\right)\right\} \leq \sup _{\left\{x \in X, \varepsilon>0: e^{h(x)} \leq M_{0}\right\}}\left\{\left(e^{h(x)}-\varepsilon\right) \gamma\left(G_{e^{h(x)}, \varepsilon}\right)\right\}$, which is achieved exactly as in Theorem 3.1 .

The following corollary gives sufficient conditions much weaker than large deviations with tight rate function in order to have the conclusions of Varadhan's theorem; in fact, we will see in the next section (Corollary 4.2) that when $X$ is normal, the condition (3.25) is also necessary.

Corollary 3.4. Let $l$ be $a[0,+\infty]$-valued function on $X$ satisfying

$$
\begin{gathered}
\lim \sup \mu_{\alpha}^{t_{\alpha}}(F) \leq \sup _{x \in G} e^{-l(x)} \leq \liminf \mu_{\alpha}^{t_{\alpha}}(G) \\
\text { (resp. } \left.\limsup \mu_{\alpha}^{t_{\alpha}}(F) \leq \sup _{x \in F} e^{-l(x)} \leq \liminf \mu_{\alpha}^{t_{\alpha}}(G)\right)
\end{gathered}
$$

for all $F \in \mathcal{F}, G \in \mathcal{G}$ with $F \subset G$. Then, $\Lambda(h)$ exists and

$$
\Lambda(h)=\sup _{x \in X, h(x)<\infty}\{h(x)-l(x)\}
$$


for all $[-\infty,+\infty]$-valued continuous functions $h$ on $X$ satisfying (3.1).

Proof. Let $h$ be a $[-\infty,+\infty]$-valued continuous function on $X$ satisfying (3.1). If (3.25) holds, then by Theorem 3.3 (with $\gamma(G)=\sup _{x \in G} e^{-l(x)}$ for all $\left.G \in \mathcal{G}\right), \Lambda(h)$ exists and

$$
e^{\Lambda(h)}=\sup _{\lambda \geq 0, \varepsilon>0}\left\{(\lambda-\varepsilon) \sup _{x \in G_{\lambda, \varepsilon}^{h}} e^{-l(x)}\right\} .
$$

Since for all $\lambda \geq 0, \varepsilon>0$ and $x \in G_{\lambda, \varepsilon}^{h}$,

$$
(\lambda-\varepsilon) e^{-l(x)} \leq e^{h(x)} e^{-l(x)},
$$

we obtain

$$
\begin{gathered}
(\lambda-\varepsilon) e^{-l(x)} \leq \sup _{x \in X, h(x)<\infty} e^{h(x)} e^{-l(x)}, \\
(\lambda-\varepsilon) \sup _{x \in G_{\lambda, \varepsilon}^{h}} e^{-l(x)} \leq \sup _{x \in X, h(x)<\infty} e^{h(x)} e^{-l(x),}
\end{gathered}
$$

and thus

$$
e^{\Lambda(h)} \leq \sup _{x \in X, h(x)<\infty} e^{h(x)} e^{-l(x)} .
$$

For all $x \in X$ with $h(x)<\infty$, and for all $\varepsilon>0$ we have

$$
\left(e^{h(x)}-\varepsilon\right) e^{-l(x)} \leq\left(e^{h(x)}-\varepsilon\right) \sup _{y \in G_{e^{h(x), \varepsilon}}^{h}} e^{-l(y)},
$$

which implies

$$
\begin{gathered}
\left(e^{h(x)}-\varepsilon\right) e^{-l(x)} \leq e^{\Lambda(h)}, \\
e^{h(x)} e^{-l(x)} \leq e^{\Lambda(h)},
\end{gathered}
$$

and finally

$$
\sup _{x \in X, h(x)<\infty} e^{h(x)} e^{-l(x)} \leq e^{\Lambda(h)} .
$$

Thus $e^{\Lambda(h)}=\sup _{x \in X, h(x)<\infty} e^{h(x)} e^{-l(x)}$, which is equivalent to (3.27). If (3.26) holds, we conclude by applying Theorem 3.3 (with $\gamma(F)=\sup _{x \in F} e^{-l(x)}$ for all $F \in \mathcal{F}$ ), and replacing $G_{\lambda, \varepsilon}^{h}$ by $F_{\lambda, \varepsilon}^{h}$, and $G_{e^{h(x)}, \varepsilon}^{h}$ by $F_{e^{h(x)}, \varepsilon}^{h}$ in the above proof.

Remark 3.5. Let $\Gamma$ be the set of maps $\gamma: \mathcal{F} \cup \mathcal{G} \rightarrow[0,1]$ such that $\gamma(F) \leq \gamma(G)$ for all $F \in \mathcal{F}, G \in \mathcal{G}$ with $F \subset G$. Define the narrow topology on $\Gamma$ as the coarsest topology for which the mappings $\gamma \mapsto \gamma(Y)$ are upper semi-continuous for all $Y \in \mathcal{F}$ and lower semi-continuous for all $Y \in \mathcal{G}$. The net $\left(\mu_{\alpha}^{t_{\alpha}}\left(\cdot{ }^{1 / t_{\alpha}}\right)\right)$ can be seen as a net in $\Gamma$ provided with the narrow topology, as well as a net in $\left[0, \infty\left[\left\{e^{h}: h \in \mathcal{C}_{b}(X)\right\}\right.\right.$ provided with the product topology. Then, the implication $(i i) \Rightarrow(i)$ in Theorem 3.3 means that if $\left(\mu_{\alpha}^{t_{\alpha}}\left(.{ }^{1 / t_{\alpha}}\right)\right)$ has a limit in $\Gamma$, then $\left(\mu_{\alpha}^{t_{\alpha}}\left(\cdot{ }^{1 / t_{\alpha}}\right)\right)$ has a limit in $\left[0, \infty\left[e^{h}: h \in \mathcal{C}_{b}(X)\right\}\right.$; moreover, the converse holds if $X$ is normal. Of course, the limit in $\Gamma$ when it exists is not unique: for each $F \in \mathcal{F}$ and $G \in \mathcal{G}, \gamma$ defined by $\gamma(F)=\limsup \mu_{\alpha}^{t_{\alpha}}(F)$ and $\gamma(G)=\liminf \mu_{\alpha}^{t_{\alpha}}(G)$ is an example, and $\gamma^{\prime}$ defined by $\gamma^{\prime}(G)=\gamma(G)$ and $\gamma^{\prime}(F)=\inf _{G \supset F, G \in \mathcal{G}} \gamma(G)$ is another one. 


\section{Criteria of A large Deviation principle}

In this section, we investigate what has to be added to the existence of $\Lambda(h)$ for all $h \in \mathcal{C}_{b}(X)$ (in other words, of the limit $\Lambda(\cdot)$ of $\left(\log \mu_{\alpha}^{t_{\alpha}}\left(e^{\cdot / t_{\alpha}}\right)\right)$ in $]-\infty,+\infty\left[\mathcal{C}_{b}(X)\right)$ in order to have large deviations. Of course, some hypotheses on $X$ are required to have sufficiently continuous functions; so we suppose here that $X$ is normal. In this case, by Theorem 3.3 (and Remark 3.5) the existence of $\Lambda(\cdot)$ on $\mathcal{C}_{b}(X)$ is equivalent to the existence of a narrow set-theoretic limit $\gamma \in \Gamma$ of $\left(\mu_{\alpha}^{t_{\alpha}}\right)$, which is also equivalent to the existence of $\Lambda(h)$ for all $[-\infty,+\infty]$-valued continuous functions $h$ on $X$ satisfying the tail condition (3.1); moreover, for each such function $h$, the variational form of $\Lambda(h)$ is given in terms of $\gamma$. In particular, $\gamma$ can vary and it is essentially this flexibility which allows us to obtain in Theorem 4.1 necessary and sufficient conditions, each of them corresponding to some type of information: a property of $\Lambda(\cdot)$ as a functional in $(i i)$, a special variational form of $\Lambda(\cdot)$ in (iii), a property of $\gamma$ in $(i v)$, and a property of the net $\left(\mu_{\alpha}^{t_{\alpha}}\right)$ in $(v)$ and $(v i)$. It is worth noticing that in both formulations (functional $(i i)$ or set-theoretic $(i v)$ ), the condition on the limit is the same: a continuity property on increasing nets. As corollaries, several basic results of the theory are strengthened by removing the tightness or compactness hypothesis.

Theorem 4.1. If $X$ is normal, then the following statements are equivalent:

(i) $\left(\mu_{\alpha}\right)$ satisfies a large deviation principle with powers $\left(t_{\alpha}\right)$.

(ii) $\Lambda(h)$ exists for all $h \in \mathcal{C}_{b}(X)$, and $\Lambda\left(h_{i}\right)$ converges to $\Lambda(h)$ for each increasing net $\left(h_{i}\right)$ in $\mathcal{C}_{b}(X)$ converging pointwise to $h \in \mathcal{C}_{b}(X)$.

(iii) $\Lambda(h)$ exists for all $h \in \mathcal{C}_{b}(X)$, and $\Lambda(h)=\sup _{x \in X}\{h(x)-l(x)\}$ for some function $l: X \rightarrow[0,+\infty]$ and for all $h \in \mathcal{C}_{b}(X)$.

(iv) There is a map $\gamma: \mathcal{G} \rightarrow[0,1]$ such that

(a) $\limsup \mu_{\alpha}^{t_{\alpha}}(F) \leq \gamma(G) \leq \liminf \mu_{\alpha}^{t_{\alpha}}(G)$ for all $F \in \mathcal{F}, G \in \mathcal{G}$ with $F \subset G$.

(b) $\gamma\left(\bigcup_{i} G_{i}\right)=\lim \gamma\left(G_{i}\right)$ for each increasing net $\left(G_{i}\right)$ in $\mathcal{G}$.

(v) $\Lambda(h)$ exists for all $h \in \mathcal{C}_{b}(X)$, and for all $F \in \mathcal{F}$, for all open covers $\left\{G_{i}\right.$ : $i \in I\}$ of $F$ and for all $\varepsilon>0$, there exists a finite subset $\left\{G_{i_{1}}, \ldots, G_{i_{N}}\right\} \subset$ $\left\{G_{i}: i \in I\right\}$ such that

$$
\liminf \mu_{\alpha}^{t_{\alpha}}(\stackrel{\circ}{F})-\limsup \mu_{\alpha}^{t_{\alpha}}\left(\bigcup_{1 \leq j \leq N} \overline{G_{i_{j}}}\right)<\varepsilon .
$$

(vi) There is a function $l: X \rightarrow[0,+\infty]$ such that

$$
\inf _{x \in F} l(x)=\sup _{G \in \mathcal{G}, G \supset F}\left\{-\liminf t_{\alpha} \log \mu_{\alpha}(G)\right\}=\sup _{G \in \mathcal{G}, G \supset F}\left\{-\lim \sup t_{\alpha} \log \mu_{\alpha}(G)\right\}
$$

for all $F \in \mathcal{F}$.

If (i) holds with rate function $J$, then the following properties hold:

$$
\begin{array}{ll}
\inf _{x \in F} J(x)=\sup _{h \in \mathcal{C}_{b}(X), h_{\mid F}=0}\{-\Lambda(h)\} & \text { for all } F \in \mathcal{F} ; \\
\inf _{x \in G} J(x)=\sup _{h \in \mathcal{C}_{b a}(X), e^{h} \leq 1_{G}}\{-\Lambda(h)\} & \text { for all } G \in \mathcal{G},
\end{array}
$$


where $C_{b a}(X)$ is the set of $[-\infty,+\infty[$-valued bounded above continuous functions on $X$; in particular,

$$
\begin{gathered}
J(x)=\sup _{h \in \mathcal{C}_{b}(X)}\{h(x)-\Lambda(h)\} \quad \text { for all } x \in X ; \\
J=\stackrel{\circ}{l} \quad \text { for all } l: X \rightarrow[0,+\infty] \text { satisfying (iii); } \\
e^{-J}(x)=\inf _{G \in \mathcal{G}, x \in G} \gamma(G)
\end{gathered}
$$

for all $x \in X$, and for all $\gamma: \mathcal{G} \rightarrow[0,1]$ satisfying $(i v)$;

$$
J=\stackrel{\circ}{l} \quad \text { for all } l: X \rightarrow[0,+\infty] \text { satisfying }(v i) .
$$

If moreover $X$ is second countable, then we can replace "net" by "sequence" in (ii) (resp. (iv)), and "open covers" by "countable open covers" in $(v)$.

Proof. $(i) \Rightarrow($ iv $)$ and (iii) $\Rightarrow$ (ii) are clear; $(i) \Rightarrow$ (iii) by Corollary 3.4 and so $(i) \Rightarrow(i i)$. If $(i)$ holds with rate function $J$, then for each $F \in \mathcal{F}$, each open cover $\left\{G_{i}: i \in I\right\}$ of $F$ and each $\varepsilon>0$,

$$
\limsup \mu_{\alpha}^{t_{\alpha}}(F) \leq \sup _{F} e^{-J} \leq \sup _{\bigcup_{i \in I} G_{i}} e^{-J}=\sup _{i \in I} \sup _{G_{i}} e^{-J}<\sup _{i \in I} \liminf \mu_{\alpha}^{t_{\alpha}}\left(G_{i}\right)+\varepsilon,
$$

which implies $(v)$.

Suppose that (ii) holds. We will prove that $(i)$ holds. Let $C_{b a}(X)$ be the set of $[-\infty,+\infty[$-valued bounded above continuous functions on $X$. By Theorem 3.3 . $\Lambda(h)$ exists in $\left[-\infty,+\infty\left[\right.\right.$ for all $h \in \mathcal{C}_{b a}(X)$, and notice that

$$
\Lambda(h \vee k)=\Lambda(h) \vee \Lambda(k)
$$

for all $k \in \mathcal{C}_{b a}(X)$; in particular,

$$
\Lambda(h \vee s)=\Lambda(h) \vee s
$$

for all $s \in\left[-\infty,+\infty\left[\right.\right.$. Let $\left(h_{i}\right)$ be an increasing net in $\mathcal{C}_{b a}(X)$ converging to $h \in \mathcal{C}_{b a}(X)$ with $\Lambda(h)>-\infty$. For each real $s<\Lambda(h)$ we have $\lim \Lambda\left(h_{i} \vee s\right)=$ $\Lambda(h \vee s)=\Lambda(h)$, and so eventually $\Lambda\left(h_{i}\right)>s$, which shows that $\lim \Lambda\left(h_{i}\right)=\Lambda(h)$. Therefore, we can replace $\mathcal{C}_{b}(X)$ by $\mathcal{C}_{b a}(X)$ in $(i i)$. Let $F \in \mathcal{F}$ and $h \in \mathcal{C}_{b a}(X)$ with $h_{\mid F}=0$. If $\Lambda(h)>-\infty$, then $\Lambda(h \vee s)=\Lambda(h)$ with $(h \vee s)_{\mid F}=0$ for all $s<\Lambda(h) \wedge 0$; if $\Lambda(h)=-\infty$, then the sequence $(\Lambda(h \vee-n))_{n \in \mathbf{N}}$ converges to $-\infty$ with $(h \vee-n)_{\mid F}=0$. Thus,

$$
\inf _{h \in \mathcal{C}_{b a}(X), h_{\mid F}=0} e^{\Lambda(h)}=\inf _{h \in \mathcal{C}_{b}(X), h_{\mid F}=0} e^{\Lambda(h)},
$$

and by the interpolation property we have

$$
\begin{aligned}
\limsup \mu_{\alpha}^{t_{\alpha}}(F) & \leq \inf _{h \in \mathcal{C}_{b a}(X), h_{\mid F}=0} e^{\Lambda(h)}=\inf _{h \in \mathcal{C}_{b}(X), h_{\mid F}=0} e^{\Lambda(h)} \\
& \leq \sup _{h \in \mathcal{C}_{b a}(X), e^{h} \leq 1_{G}} e^{\Lambda(h)} \leq \liminf \mu_{\alpha}^{t_{\alpha}}(G)
\end{aligned}
$$

for all $F \in \mathcal{F}, G \in \mathcal{G}$ with $F \subset G$. Define

$$
f(x)=\inf _{h \in \mathcal{C}_{b}(X), h(x)=0} e^{\Lambda(h)} \quad\left(=\inf _{h \in \mathcal{C}_{b a}(X), h(x)=0} e^{\Lambda(h)}\right)
$$


for all $x \in X$. By (4.9), in order to prove $(i)$ it suffices to show that $f$ is upper semi-continuous and satisfies

$$
\sup _{x \in F} f(x)=\inf _{h \in \mathcal{C}_{b}(X), h_{\mid F}=0} e^{\Lambda(h)}
$$

for all $F \in \mathcal{F}$ and

$$
\sup _{x \in G} f(x)=\sup _{h \in \mathcal{C}_{b a}(X), e^{h} \leq 1_{G}} e^{\Lambda(h)}
$$

for all $G \in \mathcal{G}$. We first show (4.10). Clearly

$$
\sup _{x \in F} f(x) \leq \inf _{h \in \mathcal{C}_{b}(X), h_{\mid F}=0} e^{\Lambda(h)}
$$

for all $F \in \mathcal{F}$. Suppose that

$$
\sup _{x \in F} f(x)<e^{s}<\inf _{h \in \mathcal{C}_{b}(X), h_{\mid F}=0} e^{\Lambda(h)}
$$

for some $F \in \mathcal{F}$ and some real $s$. Then, for all $x \in F$ there exists $h_{x} \in \mathcal{C}_{b}(X)$ which can be chosen negative such that $h_{x}(x)=0$ and

$$
\Lambda\left(h_{x}\right)<s<\inf _{h \in \mathcal{C}_{b}(X), h_{\mid F}=0} \Lambda(h) .
$$

But $1_{F} \leq e^{\bigvee_{x \in F} h_{x}}$ with $\bigvee_{x \in F} h_{x}$ bounded lower semi-continuous, and so there exists $h \in \mathcal{C}_{b}(X)$ such that $1_{F} \leq e^{h} \leq e^{\bigvee_{x \in F} h_{x}}$ (in particular $h_{\mid F}=0$ ). Let $I$ be the set of finite subsets of $F$ ordered by inclusion, and $h_{i}=h \wedge \bigvee_{x \in i} h_{x}$ for all $i \in I$, so that $\left(h_{i}\right)_{i \in I}$ is an increasing net in $\mathcal{C}_{b}(X)$ converging to $h$. Since $\Lambda\left(h_{i}\right) \leq$ $\Lambda\left(\bigvee_{x \in i} h_{x}\right)=\sup _{x \in i} \Lambda\left(h_{x}\right)<s$ for all $i \in I$, we obtain $\lim \Lambda\left(h_{i}\right)=\Lambda(h) \leq s$, which contradicts (4.12). Thus (4.10) holds. We now prove (4.11). By the interpolation property (between $1_{\{x\}}$ and $1_{G}$ ) we have clearly

$$
\sup _{x \in G} f(x) \leq \sup _{h \in \mathcal{C}_{b a}(X), e^{h} \leq 1_{G}} e^{\Lambda(h)}
$$

for all $G \in \mathcal{G}$. Suppose

$$
\sup _{x \in G} f(x)<\sup _{h \in \mathcal{C}_{b a}(X), e^{h} \leq 1_{G}} e^{\Lambda(h)}
$$

for some $G \in \mathcal{G}$. Then, for all $x \in G$ there exists $h_{x} \in \mathcal{C}_{b}(X)$ with $h_{x}(x)=0$ such that

$$
\sup _{x \in G} \Lambda\left(h_{x}\right)<s<\Lambda\left(h_{G}\right)
$$

for some $h_{G} \in \mathcal{C}_{b a}(X)$ with $e^{h_{G}} \leq 1_{G}$, and some real $s$. Let $I$ be the set of finite subsets of $G$ ordered by inclusion, and $h_{i}=h_{G} \wedge \bigvee_{x \in i} h_{x}$ for all $i \in I$, so that $\left(h_{i}\right)_{i \in I}$ is an increasing net in $\mathcal{C}_{b a}(X)$ converging to $h_{G}$. Then

$$
\Lambda\left(h_{G}\right)=\lim \Lambda\left(h_{i}\right) \leq \lim \Lambda\left(\bigvee_{x \in i} h_{x}\right)=\lim \left(\sup _{x \in i} \Lambda\left(h_{x}\right)\right) \leq s,
$$

which contradicts (4.13). Thus (4.11) holds. It remains to show that $f$ is upper semi-continuous. By (4.9), (4.10), (4.11), and since

$$
f(x)=\inf _{h \in \mathcal{C}_{b a}(X), h(x)=0} e^{\Lambda(h)} \leq \inf _{G \supset\{x\}} \sup _{h \in \mathcal{C}_{b a}(X), e^{h} \leq 1_{G}} e^{\Lambda(h)}
$$


for all $x \in X$, by Lemma 2.1 it suffices to prove that

$$
f(x)=\inf _{h \in \mathcal{C}_{b a}(X), h(x)=0} e^{\Lambda(h)} \geq \inf _{G \supset\{x\}} \sup _{h \in \mathcal{C}_{b a}(X), e^{h} \leq 1_{G}} e^{\Lambda(h)}
$$

for all $x \in X$. Suppose that (4.14) does not hold for some $x \in X$. Then, there exists $h_{x} \in \mathcal{C}_{b a}(X)$ with $h_{x}(x)=0$, and $\nu>0$ such that

$$
e^{\Lambda\left(h_{x}\right)}+\nu<\inf _{G \supset\{x\}} \sup _{h \in \mathcal{C}_{b a}(X), e^{h} \leq 1_{G}} e^{\Lambda(h)} .
$$

By (4.9) and Theorem 3.3 (with $\left.\gamma(G)=\sup _{h \in \mathcal{C}_{b a}(X), e^{h} \leq 1_{G}} e^{\Lambda(h)}\right)$, we have

$$
e^{\Lambda\left(h_{x}\right)}=\sup _{\lambda \geq 0, \varepsilon>0}\left\{(\lambda-\varepsilon) \sup _{h \in \mathcal{C}_{b a}(X), e^{h} \leq 1_{G_{\lambda, \varepsilon}^{h x}}} e^{\Lambda(h)}\right\} .
$$

Take $\lambda=1$ and $0<\varepsilon<\nu$ in (4.16), and obtain by (4.15),

$$
\sup _{h \in \mathcal{C}_{b a}(X), e^{h} \leq 1_{G_{1, \varepsilon}^{h x}}} e^{\Lambda(h)}<\inf _{G \supset\{x\}} \sup _{h \in \mathcal{C}_{b a}(X), e^{h} \leq 1_{G}} e^{\Lambda(h)}
$$

with $x \in G_{1, \varepsilon}^{h_{x}}$, which gives the contradiction. Thus (4.14) holds and $f$ is upper semi-continuous.

We have proved $(i) \Leftrightarrow(i i)$, and that when $(i)$ holds with rate function $J$, then (4.3) and (4.4) hold (by the uniqueness of a rate function on regular spaces); since

$$
\Lambda(h-h(x))=\Lambda(h)-h(x),
$$

(4.5) follows from (4.3).

Suppose that (iii) holds with $l: X \rightarrow[0,+\infty]$. Then, obviously (ii) and so $(i)$ hold; let $J$ be the associated rate function. By Corollary 3.4

$$
e^{\Lambda(h)}=\sup _{X} e^{h} e^{-J}
$$

for all $h \in C_{b a}(X)$, and so

$$
\sup _{X} e^{h} e^{-J}=\sup _{X} e^{h} e^{-l}
$$

for all $h \in C_{b}(X)$. Clearly, for each $h \in C_{b a}(X)$ there exists a real $s$ such that

$$
\sup _{X} e^{h} e^{-J}=\sup _{X} e^{h \vee s} e^{-J}
$$

and

$$
\sup _{X} e^{h} e^{-l}=\sup _{X} e^{h \vee s} e^{-l}
$$

and so by 4.18,

$$
\sup _{X} e^{h} e^{-J}=\sup _{X} e^{h} e^{-l} .
$$

For any $G \in \mathcal{G}$ choose an increasing net $\left(h_{i}\right)$ in $C_{b a}(X)$ such that $\sup _{i} e^{h_{i}}=1_{G}$, and obtain by (4.19),

$$
\sup _{G} e^{-J}=\sup _{G} e^{-l}
$$

Since $\sup _{G} e^{-l}=\sup _{G} e^{-i}$ by Lemma 2.1 we have $\sup _{G} e^{-J}=\sup _{G} e^{-i}$ for all $G \in \mathcal{G}$. Since $e^{-J}$ and $e^{-i}$ are upper semi-continuous, we have $J=\stackrel{\circ}{l}$ by Lemma 2.1. Thus, if $(i)$ holds with rate function $J$, then $J=\stackrel{\circ}{l}$ for all $l: X \rightarrow[0,+\infty]$ satisfying (iii) and (4.6) holds. 
Suppose that $(i v)$ holds with $\gamma: \mathcal{G} \rightarrow[0,1]$. Define $\gamma(F)=\inf _{G \supset F} \gamma(G)$ for all $F \in \mathcal{F}$, and notice that $\gamma$ is increasing on $\mathcal{F}$, satisfies $\gamma(F) \leq \gamma(G)$ for all $F \in \mathcal{F}$, $G \in \mathcal{G}$ with $F \subset G$, and by $(a)$,

$$
\gamma\left(\bigcup_{1 \leq j \leq N} G_{j}\right) \leq \sup _{1 \leq j \leq N} \gamma\left(\overline{G_{j}}\right)
$$

for each finite family $\left\{G_{j}\right\}_{1 \leq j \leq N} \subset \mathcal{G}$. By Theorem (3.3), $\Lambda(h)$ exists for all $h \in \mathcal{C}_{b a}(X)$ and

$$
\begin{aligned}
e^{\Lambda(h)}=\sup _{\lambda \geq 0, \varepsilon>0}\left\{(\lambda-\varepsilon) \gamma\left(G_{\lambda, \varepsilon}^{h}\right)\right\} & =\sup _{x \in X, \varepsilon>0}\left\{\left(e^{h(x)}-\varepsilon\right) \gamma\left(G_{e^{h(x), \varepsilon}}^{h}\right)\right\} \\
& =\sup _{\lambda \geq 0, \varepsilon>0}\left\{(\lambda-\varepsilon) \gamma\left(F_{\lambda, \varepsilon}^{h}\right)\right\} .
\end{aligned}
$$

We will show that $(i i)$ holds. Let $\left(h_{i}\right)_{i \in I}$ be an increasing net in $\mathcal{C}_{b}(X)$ converging to $h \in \mathcal{C}_{b}(X)$, and suppose that $\Lambda(h)>\sup _{i \in I} \Lambda\left(h_{i}\right)$. By (4.21) and (4.22), there exists $\lambda_{0} \geq 0$ and $\varepsilon_{0}>0$ such that

$$
\begin{aligned}
\left(\lambda_{0}-\varepsilon_{0}\right) \gamma\left(G_{\lambda_{0}, \varepsilon_{0}}^{h}\right) & >\sup _{i \in I} \sup _{\lambda \geq 0, \varepsilon>0}\left\{(\lambda-\varepsilon) \gamma\left(F_{\lambda, \varepsilon}^{h_{i}}\right)\right\} \\
& \geq \sup _{i \in I}\left\{\left(\lambda_{0}-\varepsilon_{0}\right) \gamma\left(F_{\lambda_{0}, \varepsilon_{0}}^{h_{i}}\right)\right\},
\end{aligned}
$$

and thus

$$
\gamma\left(G_{\lambda_{0}, \varepsilon_{0}}^{h}\right)>\sup _{i \in I} \gamma\left(F_{\lambda_{0}, \varepsilon_{0}}^{h_{i}}\right) \geq \sup _{i \in I} \gamma\left(\overline{G_{\lambda_{0}, \varepsilon_{0}}^{h_{i}}}\right) .
$$

Let $\wp$ be the set of finite subsets of $I$ ordered by inclusion, and obtain by (4.20),

$$
\forall \beta \in \wp, \quad \gamma\left(G_{\lambda_{0}, \varepsilon_{0}}^{h}\right)>\sup _{\beta \in \wp} \gamma\left(\bigcup_{i \in \beta} G_{\lambda_{0}, \varepsilon_{0}}^{h_{i}}\right) .
$$

But $G_{\lambda_{0}, \varepsilon_{0}}^{h} \subset \sup _{\beta \in \wp} \bigcup_{i \in \beta} G_{\lambda_{0}, \varepsilon_{0}}^{h_{i}}$, and the condition $(b)$ contradicts (4.23). It follows that $\Lambda(h)=\sup _{i \in I} \Lambda\left(h_{i}\right)$, that is, $(i i)$ and so $(i)$ hold; let $J$ be the associated rate function. We now prove that (4.7) holds. Let $G \in \mathcal{G}$ and $h \in C_{b a}(X)$ be such that $e^{h} \leq 1_{G}$. For all $x \in X$ and $\varepsilon>0$ with $e^{h(x)}>\varepsilon$, we have

$$
F_{e^{h(x), \varepsilon}}^{h} \subset G
$$

and

$$
\left(e^{h(x)}-\varepsilon\right) \gamma\left(F_{e^{h(x)}, \varepsilon}^{h}\right) \leq\left(e^{h(x)}-\varepsilon\right) \gamma(G) .
$$

Thus,

$$
\begin{aligned}
\sup _{\left\{x \in X ; e^{h(x)}>\varepsilon\right\}, \varepsilon>0}\left\{\left(e^{h(x)}-\varepsilon\right) \gamma\left(F_{e^{h(x)}, \varepsilon}^{h}\right)\right\} & \leq \sup _{\left\{x \in X ;, e^{h(x)}>\varepsilon\right\}, \varepsilon>0}\left\{\left(e^{h(x)}-\varepsilon\right) \gamma(G)\right\} \\
& \leq \gamma(G),
\end{aligned}
$$

and since if $e^{\Lambda(h)}>0$,

$$
e^{\Lambda(h)}=\sup _{\left\{x \in X ; e^{h(x)}>\varepsilon\right\}, \varepsilon>0}\left\{\left(e^{h(x)}-\varepsilon\right) \gamma\left(F_{e^{h(x)}, \varepsilon}^{h}\right)\right\},
$$

by (4.24) and 4.25) we obtain

$$
\sup _{h \in \mathcal{C}_{b a}(X), e^{h} \leq 1_{G}} e^{\Lambda(h)} \leq \gamma(G) .
$$


Suppose that

$$
\sup _{h \in \mathcal{C}_{b a}(X), e^{h} \leq 1_{G}} e^{\Lambda(h)}+\nu<\gamma(G)
$$

for some $\nu>0$. By taking $\lambda=1$ and $0<\varepsilon_{0}<\nu / 2$ in (4.22) we obtain

$$
\gamma\left(F_{1, \varepsilon_{0}}^{h}\right)+\nu / 2<\gamma(G)
$$

for all $h \in \mathcal{C}_{b a}(X)$ such that $e^{h} \leq 1_{G}$. Let $\left(h_{i}\right)$ be an increasing net in $\mathcal{C}_{b a}(X)$ such that $\sup _{i} e^{h_{i}}=1_{G}$, and let $\wp$ be the set of finite subsets of $I$ ordered by inclusion. Then $\left(\bigcup_{i \in \beta} G_{1, \varepsilon_{0}}^{h_{i}}\right)_{\beta \in \wp}$ is an increasing net in $\mathcal{G}$ such that

$$
\forall \varepsilon>0, \quad G \subset \bigcup_{\beta \in \wp} \bigcup_{i \in \beta} G_{1, \varepsilon_{0}}^{h_{i}} .
$$

By (4.27) we have

$$
\forall i \in I, \quad \gamma\left(\overline{G_{1, \varepsilon_{0}}^{h_{i}}}\right)+\nu / 2 \leq \gamma\left(F_{1, \varepsilon_{0}}^{h_{i}}\right)+\nu / 2<\gamma(G),
$$

and by (4.20),

$$
\forall \beta \in \wp, \quad \gamma\left(\bigcup_{i \in \beta} G_{1, \varepsilon_{0}}^{h_{i}}\right)+\nu / 2<\gamma(G),
$$

which contradicts (4.28) by (b). Therefore,

$$
\sup _{h \in \mathcal{C}_{b a}(X), e^{h} \leq 1_{G}} e^{\Lambda(h)}=\gamma(G),
$$

and by (4.4)

$$
\sup _{G} e^{-J}=\gamma(G),
$$

which gives (4.7) by upper semi-continuity of $e^{-J}$.

Suppose that $(v)$ holds. By Theorem 3.3,

$$
e^{\Lambda(h)}=\sup _{\lambda \geq 0, \varepsilon>0}\left\{(\lambda-\varepsilon) \liminf \mu_{\alpha}^{t_{\alpha}}\left(G_{\lambda, \varepsilon}^{h}\right)\right\}=\sup _{\lambda \geq 0, \varepsilon>0}\left\{(\lambda-\varepsilon) \limsup \mu_{\alpha}^{t_{\alpha}}\left(F_{\lambda, \varepsilon}^{h}\right)\right\}
$$

for all $h \in \mathcal{C}_{b}(X)$. Let $\left(h_{i}\right)_{i \in I}$ be an increasing net in $\mathcal{C}_{b}(X)$ converging to $h \in \mathcal{C}_{b}(X)$. We will prove that $\lim \Lambda\left(h_{i}\right)=\Lambda(h)$. If $\Lambda(h)>\sup _{i \in I} \Lambda\left(h_{i}\right)$, then there exists $\lambda_{0} \geq 0, \varepsilon_{0}>0$ and $\nu>0$ such that

$$
\begin{aligned}
\left(\lambda_{0}-\varepsilon_{0}\right) \liminf \mu_{\alpha}^{t_{\alpha}}\left(G_{\lambda_{0}, \varepsilon_{0}}^{h}\right) & >\sup _{i \in I} \sup _{\lambda \geq 0, \varepsilon>0}\left\{(\lambda-\varepsilon) \limsup \mu_{\alpha}^{t_{\alpha}}\left(F_{\lambda, \varepsilon}^{h_{i}}\right)\right\}+\nu \\
& \geq \sup _{i \in I} \sup _{\lambda \geq 0, \varepsilon>0}\left\{(\lambda-\varepsilon) \limsup \mu_{\alpha}^{t_{\alpha}}\left(\overline{G_{\lambda, \varepsilon}^{h_{i}}}\right)\right\}+\nu \\
& \geq \sup _{i \in I} \sup _{\varepsilon>0}\left\{\left(\lambda_{0}-\varepsilon+\nu / 2\right) \limsup \mu_{\alpha}^{t_{\alpha}}\left(\overline{G_{\lambda_{0}, \varepsilon}^{h_{i}}}\right)\right\}+\nu / 2 .
\end{aligned}
$$

Take $\varepsilon_{0}<\varepsilon<\varepsilon_{0}+\nu / 2$ in (4.29) and obtain

$$
\left(\lambda_{0}-\varepsilon_{0}\right) \liminf \mu_{\alpha}^{t_{\alpha}}\left(G_{\lambda_{0}, \varepsilon_{0}}^{h}\right)>\left(\lambda_{0}-\varepsilon_{0}\right) \sup _{i \in I} \limsup \mu_{\alpha}^{t_{\alpha}}\left(\overline{G_{\lambda_{0}, \varepsilon}^{h_{i}}}\right)+\nu / 2 .
$$

Put $F=F_{\lambda_{0}, \varepsilon_{0}}^{h}, G_{i}=G_{\lambda_{0}, \varepsilon}^{h_{i}}$ for all $i \in I$, and notice that $F \subset \bigcup_{i \in I} G_{i}$. Since $\stackrel{\circ}{F} \supset G_{\lambda_{0}, \varepsilon_{0}}^{h}$ we obtain by (4.30),

$$
\liminf \mu_{\alpha}^{t_{\alpha}}(\stackrel{\circ}{F})>\sup _{i \in I} \lim \sup \mu_{\alpha}^{t_{\alpha}}\left(\overline{G_{i}}\right)+\nu / 2,
$$


and so

$$
\liminf \mu_{\alpha}^{t_{\alpha}}(\stackrel{\circ}{F})>\limsup \mu_{\alpha}^{t_{\alpha}}\left(\bigcup_{1 \leq j \leq N} \overline{G_{i_{j}}}\right)+\nu / 2,
$$

for all finite subsets $\left\{G_{i_{j}}: 1 \leq j \leq N\right\} \subset\left\{G_{i}: i \in I\right\}$, which contradicts (4.1). Thus $\lim \Lambda\left(h_{i}\right)=\Lambda(h)$, that is, $(i i)$ and so $(i)$ hold.

It remains to prove $(i) \Leftrightarrow(v i)$, (4.8) and the last assertion. Suppose that $(i)$ holds with rate function $J$. Put $f=e^{-J}$ and let $F \in \mathcal{F}$. By (4.3) we have

$\sup _{x \in F} f(x)=\inf _{h \in \mathcal{C}_{b}(X), h_{\mid F}=0} e^{\Lambda(h)} \leq \inf _{G \in \mathcal{G}, G \supset F} \liminf \mu_{\alpha}^{t_{\alpha}}(G) \leq \inf _{G \in \mathcal{G}, G \supset F} \lim \sup \mu_{\alpha}^{t_{\alpha}}(G)$.

Suppose that

$$
\inf _{h \in \mathcal{C}_{b}(X), h_{\mid F}=0} e^{\Lambda(h)}<\inf _{G \in \mathcal{G}, G \supset F} \lim \sup \mu_{\alpha}^{t_{\alpha}}(G) .
$$

Then, there exists $\nu>0$ and $h_{F} \in \mathcal{C}_{b}(X)$ with $h_{F \mid F}=0$ such that

$$
e^{\Lambda\left(h_{F}\right)}+\nu<\inf _{G \in \mathcal{G}, G \supset F} \lim \sup \mu_{\alpha}^{t_{\alpha}}(G) .
$$

Since

$$
\left.e^{\Lambda\left(h_{F}\right)}=\sup _{\left\{x \in X ; h_{F}(x)<\infty\right\}, \varepsilon>0}\left\{\left(e^{h_{F}(x)}-\varepsilon\right) \limsup \mu_{\alpha}^{t_{\alpha}}\left(F_{e^{h_{F}(x)}, \varepsilon}^{h_{F}}\right)\right\}\right)
$$

by Theorem 3.3, by taking $\varepsilon=\varepsilon_{0}<\nu$ in (4.32) we obtain by (4.31),

$$
\left.\sup _{\left\{x \in X ; h_{F}(x)<\infty\right\}}\left\{e^{h_{F}(x)} \limsup \mu_{\alpha}^{t_{\alpha}}\left(F_{e^{h_{F}(x)}, \varepsilon_{0}}^{h_{F}}\right)\right\}\right)<\inf _{G \in \mathcal{G}, G \supset F} \limsup \mu_{\alpha}^{t_{\alpha}}(G) .
$$

Since $h_{F}(x)=0$ for all $x \in F$, we have $F \subset G_{1, \varepsilon_{0}}^{h_{F}} \subset F_{1, \varepsilon_{0}}^{h_{F}}$ and (4.33) implies

$$
\limsup \mu_{\alpha}^{t_{\alpha}}\left(G_{1, \varepsilon_{0}}^{h_{F}}\right) \leq \limsup \mu_{\alpha}^{t_{\alpha}}\left(F_{1, \varepsilon_{0}}^{h_{F}}\right)<\inf _{G \in \mathcal{G}, G \supset F} \lim \sup \mu_{\alpha}^{t_{\alpha}}(G)
$$

and the contradiction. We have shown that

$$
\sup _{x \in F} f(x)=\inf _{G \in \mathcal{G}, G \supset F} \liminf \mu_{\alpha}^{t_{\alpha}}(G)=\inf _{G \in \mathcal{G}, G \supset F} \lim \sup \mu_{\alpha}^{t_{\alpha}}(G) \quad \text { for all } F \in \mathcal{F},
$$

which is equivalent to (4.2) with $l=J$, and so (vi) holds.

If $(v i)$ holds with $l: X \rightarrow[0,+\infty]$, then (4.2) implies

$$
\limsup \mu_{\alpha}^{t_{\alpha}}(F) \leq \sup _{F} e^{-l} \leq \liminf \mu_{\alpha}^{t_{\alpha}}(G)
$$

for all $F \in \mathcal{F}, G \in \mathcal{G}$ with $F \subset G$. By Proposition 2.3, (i) holds with rate function $\stackrel{\circ}{l}$ and $(4.8)$ holds.

If moreover $X$ is second countable, then it is well known that for any family $\left\{h_{i}: i \in I\right\}$ of lower semi-continuous functions on $X$ there exists a countable subset $I_{0} \subset I$ such that $\sup _{i \in I} h_{i}=\sup _{i \in I_{0}} h_{i}$. It is easy to see in the above proof that this property allows us to replace "net" by "sequence" in (ii) (resp. (iv)), and "open covers" by "countable open covers" in $(v)$.

By combining Theorem 4.1 with Corollary 3.4 and Lemma 2.1, we obtain in the following corollary necessary and sufficient conditions in order that a large deviation principle occurs with rate function the lower regularization $l$ of a given function $l: X \rightarrow[0,+\infty]$. Notice that by Proposition 2.3 and $(i) \Leftrightarrow($ ii $)$ in Corollary 4.2 the infimum of the set of $[0,+\infty]$-valued functions $l$ on $X$ satisfying (3.25) coincides with the lower regularization $l$ of each its elements. The equivalence $(i) \Leftrightarrow($ iii $)$ in 
Corollary 4.2 was known when $l$ is a tight rate function ([2], Theorem 4.4.13); here there is no hypothesis on $l$.

Corollary 4.2. Suppose that $X$ is normal, and let $l$ be $a[0,+\infty]$-valued function on $X$. Then, the following statements are equivalent:

(i) $\left(\mu_{\alpha}\right)$ satisfies a large deviation principle with powers $\left(t_{\alpha}\right)$ and rate function $\stackrel{\circ}{l}$.

(ii)

$$
\limsup \mu_{\alpha}^{t_{\alpha}}(F) \leq \sup _{x \in G} e^{-l(x)} \leq \liminf \mu_{\alpha}^{t_{\alpha}}(G)
$$

for all $F \in \mathcal{F}, G \in \mathcal{G}$ with $F \subset G$.

(iii) $\Lambda(h)$ exists and

$$
\Lambda(h)=\sup _{x \in X}\{h(x)-l(x)\} \quad \text { for all } h \in \mathcal{C}_{b}(X) .
$$

(iv) $\Lambda(h)$ exists and

$$
\Lambda(h)=\sup _{x \in X, h(x)<\infty}\{h(x)-l(x)\}
$$

for all $[-\infty,+\infty]$-valued continuous functions $h$ on $X$ satisfying (3.1).

Proof. (ii) $\Rightarrow$ (iv) $\Rightarrow$ (iii) by Corollary 3.4 (iii) $\Rightarrow$ (i) by Theorem 4.1, and $(i) \Rightarrow(i i)$ since $\sup _{x \in G} e^{-l(x)}=\sup _{x \in G} e^{-i(x)}$ for all $G \in \mathcal{G}$ by Lemma 2.1 .

Recall that $\left(\mu_{\alpha}\right)$ is said to be exponentially tight with respect to $\left(t_{\alpha}\right)$ if for all $\varepsilon>0$ there is a compact set $K \subset X$ such that $\lim \sup \mu_{\alpha}^{t_{\alpha}}(X \backslash K)<\varepsilon$. Bryc's theorem ([2], Theorem 4.4.2) states that if $\Lambda(h)$ exists for all $h \in \mathcal{C}_{b}(X)$ and if $\left(\mu_{\alpha}\right)$ is exponentially tight with respect to $\left(t_{\alpha}\right)$, then $\left(\mu_{\alpha}\right)$ satisfies a large deviation principle with powers $\left(t_{\alpha}\right)$; moreover, the (necessarily tight) rate function satisfies (4.5). The following Corollary 4.3 shows that the first conclusion holds under a hypothesis clearly weaker than exponential tightness. Moreover, Theorem4.1 states that without any tightness hypothesis, a rate function for $\left(\mu_{\alpha}^{t_{\alpha}}\right)$ always satisfies (4.5).

Corollary 4.3. Suppose that $X$ is normal. If $\Lambda(h)$ exists for all $h \in \mathcal{C}_{b}(X)$, and if for all open covers $\left\{G_{i}: i \in I\right\}$ of $X$, for all $\varepsilon>0$, there exists a finite subset $\left\{G_{i_{1}}, \ldots, G_{i_{N}}\right\} \subset\left\{G_{i}: i \in I\right\}$ such that

$$
\limsup \mu_{\alpha}^{t_{\alpha}}\left(X \backslash \bigcup_{1 \leq j \leq N} \overline{G_{i_{j}}}\right)<\varepsilon,
$$

then $\left(\mu_{\alpha}\right)$ satisfies a large deviation principle with powers $\left(t_{\alpha}\right)$.

Proof. Let $F \in \mathcal{F},\left\{G_{i}: i \in I\right\}$ be an open cover of $F$ and $\varepsilon>0$. Then, $\bigcup_{i \in I} G_{i} \cup$ $X \backslash F$ is an open cover of $X$, and so there exists a finite subset $\left\{G_{i_{1}}, \ldots, G_{i_{N}}\right\} \subset$ $\left\{G_{i}: i \in I\right\}$ such that

$$
\limsup \mu_{\alpha}^{t_{\alpha}}\left(X \backslash\left(\bigcup_{1 \leq j \leq N} \overline{G_{i_{j}}} \cup X \backslash \stackrel{\circ}{F}\right)\right)<\varepsilon
$$

Thus,

$$
\limsup \mu_{\alpha}^{t_{\alpha}}\left(\stackrel{\circ}{F} \backslash \bigcup_{1 \leq j \leq N} \overline{G_{i_{j}}}\right)<\varepsilon
$$

and by Theorem 4.1 the conclusion holds. 
Corollary 4.4. If $X$ is normal and $\left(\mu_{\alpha}^{t_{\alpha}}\right)$ satisfies (4.34), then $\left(\mu_{\alpha}\right)$ has a subnet $\left(\mu_{\beta}\right)$ satisfying a large deviation principle with powers $\left(t_{\beta}\right)$.

Proof. Define $\Lambda_{\alpha}(h)=\log \mu_{\alpha}^{t_{\alpha}}\left(e^{h / t_{\alpha}}\right)$ for all $h \in \mathcal{C}_{b}(X)$. Then, $\left(\Lambda_{\alpha}(\cdot)\right)$ is a net in the compact space $[-\infty,+\infty]^{\mathcal{C}_{b}(X)}$ (with the product topology), and so there is a subnet $\left(\Lambda_{\beta}(\cdot)\right)$ converging to some limit $\Lambda^{\prime}(\cdot)$. The result follows from Corollary 4.3 applied to $\left(\mu_{\beta}^{t_{\beta}}\right)$.

Remark 4.5. $(i) \Leftrightarrow(i v)$ in Theorem 4.1 was known when $\gamma$ is a sup-preserving capacity in the O'Brien sense, i.e., $\gamma(G)=\sup \{\gamma(K): K \subset G, K$ compact $\}$ for all $G \in$ $\mathcal{G}, \gamma(K)=\inf \{\gamma(G): G \supset K, G \in \mathcal{G}\}$ for all compact $K \subset X$, and $\gamma$ satisfies (2.1) of Lemma 2.1 (3). Thus, Theorem 4.1 removes the sup-preserving as well as the capacity conditions of $\gamma$ (notice the difference between $(i v)$ in Theorem 4.1 and (ii) in Proposition 2.3). In the spirit of Remark 3.5 this means that $\left(\mu_{\alpha}\right)$ satisfies a large deviation principle with powers $\left(t_{\alpha}\right)$ if and only if $\left(\mu_{\alpha}^{t_{\alpha}}\right)$ has a narrow set-theoretic limit in the set $\left\{\gamma \in \Gamma: \lim \gamma\left(G_{i}\right)=\gamma\left(\bigcup_{i} G_{i}\right)\right.$ for all increasing nets $\left(G_{i}\right)$ in $\left.\mathcal{G}\right\}$.

Remark 4.6. When $X$ is Polish and $\left(\mu_{\alpha}\right)=\left(\mu_{n}\right)_{n \in \mathbf{N}_{*}}$, a recent result of Bryc and Bell ([1], Theorem 2.1) implies the equivalence of the following statements:

(i') $\left(\mu_{n}\right)$ satisfies a large deviation principle with powers $(1 / n)$ and tight rate function;

(ii') $\Lambda(h)$ exists for all $h \in C_{b}(X)$, and $\inf _{m} \Lambda\left(h_{m}\right)=\Lambda(h)$ for each decreasing sequence $\left(h_{m}\right)$ in $C_{b}(X)$ converging to $h \in C_{b}(X)$.

The equivalence $(i) \Leftrightarrow($ ii $)$ together with the last assertion in Theorem 4.1 can be seen as a free tightness analogue of that, by replacing "decreasing" by "increasing" in (ii'), and removing "tight" in $\left(\mathrm{i}^{\prime}\right)$.

Remark 4.7. The relation (4.2) in Theorem 4.1 generalizes a well-known expression of a rate function $J$ for $\left(\mu_{\alpha}^{t_{\alpha}}\right)$, obtained with $l=J$ and $F$ ranging over all singletons ([2], Theorem 4.1.18).

\section{REFERENCES}

1. W. Bryc and H. Bell. Variational representations of Varadhan functionals, Proc. Amer. Math. Soc., 129 (2001), No. 7, pp. 2119-2125. MR 2002b:60040

2. A. Dembo and O. Zeitouni. Large deviations techniques and applications, Second edition, Springer-Verlag, New York, 1998. MR 99d:60030

3. G. L. O'Brien and W. Verwaat. Capacities, large deviations and loglog laws. Stable Processes and Related Topics (Ithaca, NY, 1990), pp. 43-83, Progr. Probab. 25, Birkhäuser, Boston, MA, 1991. MR 92k:60007

Department of Mathematics, University of Santiago of Chile, Bernardo O'Higgins 3363, Santiago, Chile

E-mail address: hcomman@usach.cl 